\title{
Applications of superconducting magnetic energy storage in electrical power systems
}

\author{
S BANERJEE and T N SAHA \\ Department of Electrical Engineering, Indian Institute of Technology, Kharagpur 721 302, \\ India
}

\begin{abstract}
Fast-acting energy storage devices can effectively damp electromechanical oscillations in a power system, because they provide storage capacity in addition to the kinetic energy of the generator rotor, which can share the sudden changes in power requirement. The present paper explores the means of reducing the inductor size for this application so that the use of high- $T_{c}$ superconducting materials becomes feasible.
\end{abstract}

Keywords. Magnetic energy storage; electrical power systems.

\section{Introduction}

Superconducting magnetic energy storage (SMES) system has numerous advantages in electrical power system applications over other conventional means of electrical energy storage, like pumped hydro energy storage, compressed air energy storage tc. Apart from being the most efficient, SMES has attracted the attention of power engineers in recent years because of its extremely fast response in switching over from charging mode to discharging mode and the high rate of energy discharge capability. The other means of electrical energy storage are inherently handicapped on these two counts.

Recognizing these advantages the Bonneville Power Administration of USA has already installed a $30 \mathrm{MJ}$ SMES unit to stabilize the Pacific AC Intertie that connects the Northern and Southern power systems of that country (Rogers et al 1979, 1983; Boenig and Hauer 1985; Hassenzahl 1983). Detailed designs of massive SMES installations (of the order of $1 \mathrm{GWH}$ ) for load-levelling and peak shaving purposes have been evolved (Rogers et al 1979; Loyd 1986). However, the high cost of low temperature metallic superconductor technology has so far inhibited the wide-scale application of superconducting magnets in power systems.

With the discovery of high- $T_{c}$ ceramic superconductors, the application of SMES in power system may acquire commercial viability in the near future. However, considerations of critical current density under high magnetic fields may restrict the energy storage capacity of such units.

This paper shows that SMES units with such small energy storage capacity can be used in load-frequency control of power systems. This problem occurs when there is a sudden change in load. The steam inlet to the turbine cannot change instantaneously and the additional load has to be met from the kinetic energy of the generator rotors. Consequently the rotor speed falls, the frequency drops, and normalcy can be restored only after a series of oscillations when the steam governor responds to the frequency deviation. These frequency oscillations are a persistent source of trouble in a power system and occasionally lead to instability and shut downs.

The LFC problem fundamentally being that of instantaneous mismatch between supply and demand of real power in a power system, a fast acting magnetic energy storage unit can improve the performance of a system by sharing the change in load till 
the steam governor comes into action. Since the mismatch between demand and supply of power usually lasts for 5-10 seconds, the energy storage requirement is small for this application.

This work aims at presenting a strategy for controlling the SMES unit so that the energy storage requirement of the magnet can be further reduced for the same damping action. This opens up a new area of application of the emerging ceramic superconductor technology in future.

\section{Power modulation in SMES units}

The SMES inductor-converter unit consists of a dc superconducting inductor, a 12 pulse cascaded bridge type ac/dc converter and a $Y-Y / \Delta$ step down transformer (figure 1). Control of the converter firing angle enables the dc voltage $E_{d}$ appearing across the inductor to be continuously varied between a wide range of positive and negative values. The converter dc output current $I_{d}$ being unidirectional, the control for the direction and magnitude of the inductor power flow $P_{d}$, is achieved by continuously regulating the firing angle $\alpha$.

After initial charging of the inductor, the simplest control strategy can be to control the dc voltage $E_{d}$ across the inductor depending on the sensed frequency deviation signal $\Delta f$ (Peterson et al 1975). The incremental change in the inductor voltage $\Delta E_{d}$ is given by

$$
\Delta E_{d}=\left[K_{0} /\left(1+T_{\mathrm{dc}} \cdot p\right)\right] \Delta f,
$$

where $K_{0}$ is the gain of the control loop, $T_{\mathrm{dc}}$ the converter time delay and $p$ the differential operator $\mathrm{d} / \mathrm{d} t$. When the system load increases the bus frequency falls. Consequently a negative voltage $\Delta E_{d}$, shown in (1), is impressed across the inductor. The inductor current being unidirectional, application of a negative voltage across it results in withdrawal of energy.
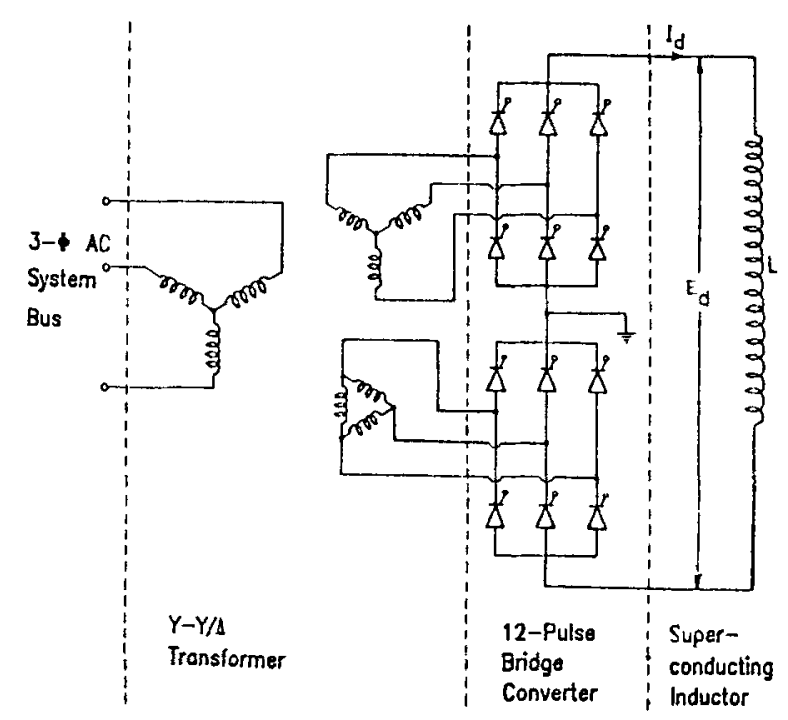

Figure 1. Schematic diagram of the magnetic energy storage unit. 
The power withdrawal and consequent deviation of inductor current are given by the following equations.

$$
\begin{aligned}
\Delta P_{d} & =I_{d 0} \cdot \Delta E_{d}+\Delta E_{d} \cdot \Delta I_{d} \\
\Delta I_{d} & =\Delta E_{d} / p \cdot L
\end{aligned}
$$

While following (1), the relationship between $\Delta E_{d}$ and $\Delta f$ has to operate within two constraints: (a) the voltage $E_{d}$ across the inductor should be within certain design limits and (b) the inductor current deviation $\Delta I_{d}$ during the disturbance should remain within specific upper and lower bounds.

\section{Effects of SMES unit on LFC loop}

In order to study the effects of SMES units in a power system, a two-area system of unequal capacities has been considered (see figure 2). Area 1 (of capacity $1000 \mathrm{MW}$ ) includes generators $G_{11}, G_{12}, \ldots G_{1 m}$ connected to a common busbar while area 2 (of capacity $3000 \mathrm{MW}$ ) includes generators $G_{21}, G_{22}, \ldots G_{2 \pi}$ connected to another common bus. Areas 1 and 2 are connected by a weak tie line. An SMES unit is connected to area 1 bus, as it is expected to experience larger load frequency disturbance due to its relatively low capacity. $P_{L 1}$ and $P_{L 2}$ are lumped initial system loads to areas 1 and 2 respectively and power system response to step load change $\Delta P_{L 1}=0.01$ p.u.MW is studied.

The investigation has been carried out by simulating the above power system on a digital computer by adding the state variable equations of the SMES unit to those of the power system (Elgerd 1983). The nominal parameters of the power system are as follows:

$$
\begin{aligned}
& P_{R 1}=1000 \mathrm{MW}, P_{R 2}=3000 \mathrm{MW}, K_{p 1}=K_{p 2}=100.0 \mathrm{~Hz} / \text { p.u.MW, } \\
& T_{p 1}=T_{p 2}=20.0 \mathrm{~s}, T_{G 1}=T_{G 2}=0.08 \mathrm{~s}, T_{T 1}=T_{T 2}=0.3 \mathrm{~s}, \\
& K_{R 1}=K_{R 2}=0.5, T_{R 1}=T_{R 2}=10.0 \mathrm{~s}, R_{1}=R_{2}=2.4 \mathrm{~Hz} / \text { p.u.MW, } \\
& B_{1}=B_{2}=0.425, T_{12}^{\circ}=0.0866, a_{12}=-0.333 .
\end{aligned}
$$

The parameters of the SMES unit are: $I_{d 0}=4.0 \mathrm{KA}, L=1.0 \mathrm{H}, T_{\mathrm{dc}}=0.026 \mathrm{~s}$, and $K_{0}=100 \mathrm{kV} / \mathrm{Hz}$ unless otherwise specified in the figures.

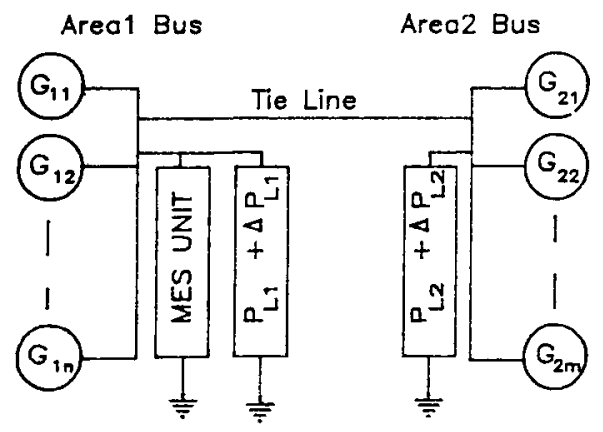

Figure 2. Single line diagram of the two-area power system. 

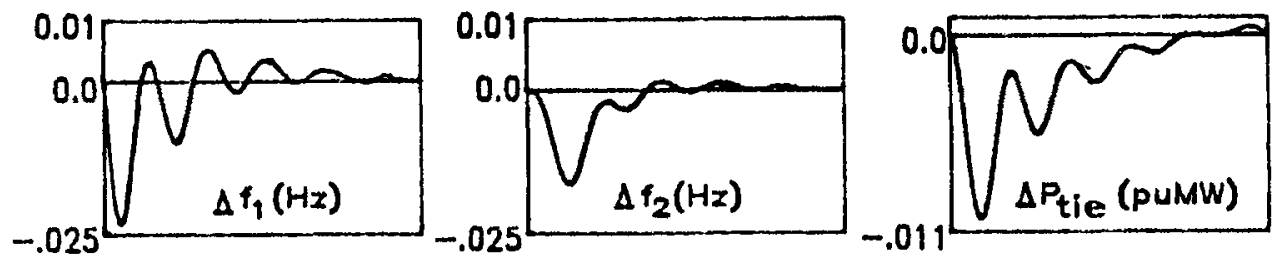

Figure 3. Response of the power system without SMES unit for $15 \mathrm{~s}$. Step disturbance $\Delta P_{L 1}=0.01$ p.u.
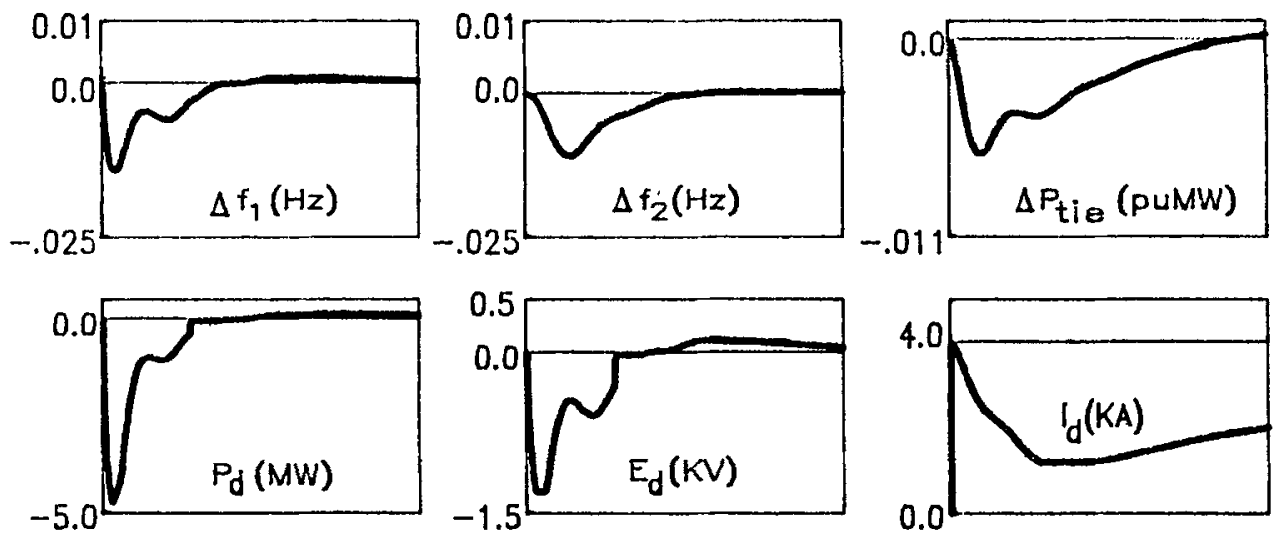

Figure 4. Response of the power system with SMES unit for $15 \mathrm{~s}$. Step disturbance $\Delta P_{L 1}=0.01$ p.u., $K_{0}=100 \mathrm{kV} / \mathrm{Hz}$.

Figure 3 shows the deviations in frequencies $\Delta f_{1}$ and $\Delta f_{2}$ and tie-line power $\Delta P_{\text {tie }}$ without the energy storage unit in response to a step load change of 0.01 p.u. in area 1. The behaviour of the power system with the SMES unit under the same condition is shown in figure 4 . It can be seen that the damping improves with the addition of the SMES unit and the maximum overshoots of $\Delta f_{1}, \Delta f_{2}$ and $\Delta P_{\text {tie }}$ reduce by approximately $40 \%, 30 \%$ and $35 \%$ respectively. Figure 4 also shows the plots of the voltage, current and power in the SMES unit.

\section{Suggested control strategy}

The first step in minimizing the inductor size is to reduce the current deviation in the inductor and quick restoration of the current to normal value following a load disturbance. It has been found that this can be achieved by using the inductor current deviation feedback in the control loop.

Secondly, in multiarea power systems it is desirable that each area carry its own load, least participating in the disturbances in the other areas. This reduces the inadvertent interchange of power between areas during frequency oscillations.

The present study has shown that in the SMES installations where the tie-line power deviation signals are available, this effect can be achieved by the use of area control error (ACE) in place of frequency deviation in the input of the SMES control logic. 

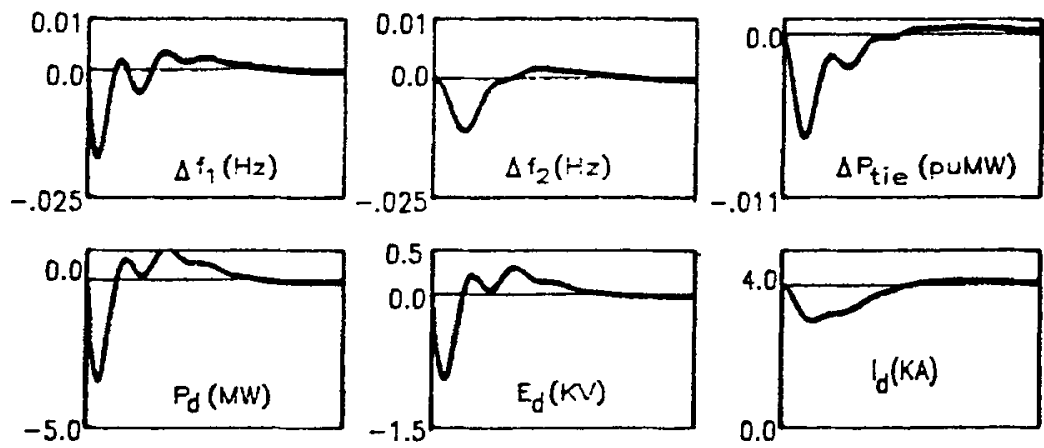

Figure 5. Response of the power system with optimized value of $K_{I}$ for $15 \mathrm{~s}$. $K_{0}=100 \mathrm{kV} / \mathrm{unit} \mathrm{ACE}, K_{I d}=0.5 \mathrm{kV} / \mathrm{KA}, K_{I}=0.81$

Thus, with the ACE input and $\Delta I_{d}$ feedback the inductor voltage deviation can be expressed as

$$
\Delta E_{d}=\frac{1}{T_{\mathrm{dc}} \cdot p+1}\left[K_{0}\left(\Delta P_{\mathrm{tic}}+B \cdot \Delta f\right)-K_{I d} \cdot \Delta I_{d}\right]
$$

where $K_{I d}$ is the gain for $\Delta I_{d}$ feedback, and $B$ the frequency bias parameter.

It may be noted that when the power system demands the extra power during the first few seconds following the disturbance, it is supplied as required. The $\left(K_{I d} \cdot \Delta I_{d}\right)$ term in the above equation becomes effective only when the inductor current has deviated by a considerable amount. Consequently, this strategy leads to better utilization of the energy storage in the inductor.

It has been found in the present work that the energy storage unit partially counteracts the effects of the integral controller associated with automatic generation control. This implies that the optimal setting of the integrator gain $K_{I}$ is altered with the addition of the SMES unit. The new optimal setting of $K_{I}$ is computed using the integral square error criterion and it has been found that the optimal integrator gain setting is altered from 0.44 to 0.81 with the addition of the SMES unit. The advantage of a higher setting of the integrator gain is in the reduction of the settling time. This reduces the duration of power flow from the inductor and consequently further reduces the energy storage requirement.

The response of the optimized system for $K_{0}=100 \mathrm{kV} / \mathrm{unit} \mathrm{ACE}, K_{I d}=0.5 \mathrm{kV} / \mathrm{kA}$, and $K_{I}=0.81$ has been shown in figure 5 . It clearly shows the improvement in transient response (with a lesser settling time) in comparison with that in figure 4.

\section{Reduction in inductor size}

From the foregoing discussion it can be concluded that the application of inductor current deviation feedback and increase of integrator gain setting reduces the energy withdrawal from the SMES unit, thus allowing the use of an inductor of much smaller inductance. Figure 6 shows the response of the power system when the inductor size is reduced from 1.0 to 0.5 Henry. It shows that an SMES unit of energy storage capacity of $4 \mathrm{MJ}$ suffices in reducing the maximum frequency and tie-line power deviations by 

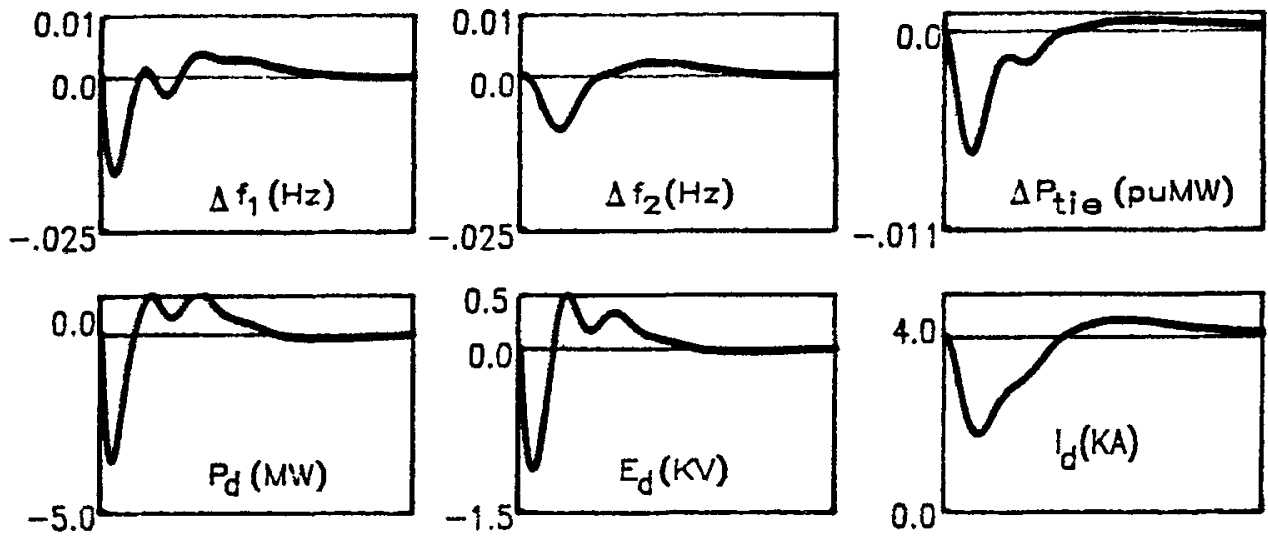

Figure 6. Response of the power system and the SMES unit when the inductance is reduced to $0.5 \mathrm{H}$. The other parameters remain the same.

approximately $40 \%$. In the present work it has also been found that for an area of capacity $2000 \mathrm{MW}$, the required energy storage capacity is of the order of $6 \mathrm{MJ}$.

\section{Conclusions}

It has been shown in the present work that small-sized SMES units with suitable control can effectively reduce the frequency and tie-line power oscillations following sudden small load perturbations.

The control logic suggested for this purpose takes the area control error as its input and uses inductor current deviation feedback. In a power system with SMES unit, the optimal setting of the integrator gain is altered to a higher value. With the suggested control measure, an SMES unit of $4 \mathrm{MJ}$ capacity can reduce the maximum deviations of frequency and tie-line power flow by approximately $40 \%$ in a $1000 \mathrm{MW}$ power area.

For the above storage capacity, high- $T_{c}$ superconducting inductors may be a viable option. However, further research effort is necessary to evolve suitable designs of such superconducting inductors in sizes required for LFC application.

\section{References}

Boenig H J and Hauer J F 1985 IEEE Trans. PAS 104302

Elgerd O I 1983 in Electric energy systems theory-An introduction (New Delhi: Tata McGraw-Hill) 2nd edn Hassenzahl W V 1983 Proc. IEEE 711089

Loyd R J 1986 IEEE Trans. on Energy Conversion, Vol. EC-1, 63

Peterson H A, Mohan N and Boom R W 1975 IEEE Trans. Power Apparatus \& Systems 941337

Rogers J D, Schermer R I, Miller B L and Hauer J F 1983 Proc. IEEE 711099

Rogers J D, Boenig H J, Bronson J C, Coyler D S, Hassenzahl W V, Turner R D and Schermer R I 1979 IEEE Trans. Mag. M15 820 